\title{
High-intensity focused ultrasound compared with irradiation for ovarian castration in premenopausal females with hormone receptor-positive breast cancer after radical mastectomy
}

\author{
SHU-WEN WANG, XIN-YING HE and MING-ZHONG LI \\ Department of Radiation Oncology, The First Affiliated Hospital of Medical College of Xi'an Jiaotong University, \\ Xi'an, Shaanxi 710061, P.R. China
}

Received May 16, 2012; Accepted July 20, 2012

DOI: $10.3892 / \mathrm{ol} .2012 .860$

\begin{abstract}
The aim of the current study was to determine the feasibility, efficacy and safety of ovarian castration by high-intensity focused ultrasound (HIFU) in premenopausal patients with estrogen receptor $(\mathrm{ER})^{+} /$progesterone receptor $(\mathrm{PR})^{+}$breast cancer subsequent to radical mastectomy. A total of 88 premenopausal females with pathologically confirmed $\mathrm{ER}^{+} / \mathrm{PR}^{+}$breast cancer following radical mastectomy were randomly and equally divided into two groups that received HIFU therapy or radiation treatment. HIFU therapy was applied twice at an interval of three days and radiotherapy was administered to a total prescribed dose of $\mathrm{D}_{\mathrm{T}} 18 \mathrm{~Gy}$ in nine fractions over 11 days. Outcome measures included serum levels of estradiol and estrone, the Kupperman index and the incidence of secondary amenorrhea. Adverse events were monitored and recorded. All patients were followed up for 12 months. Serum levels of estradiol and estrone were comparable prior to treatment between the HIFU and radiation treatment groups. One month following treatment, serum levels of estradiol and estrone were significantly decreased in the two groups, but a greater decline was observed in the HIFU treatment group ( $\mathrm{P}<0.01$ and 0.05 , respectively). In addition, more patients developed severe menopausal symptoms and amenorrhea in the HIFU therapy group compared with the radiotherapy group ( $\mathrm{P}<0.01$ for the two groups). A total of 3 months following treatment, serum levels of estradiol and estrone and the distribution of patients with severe, moderate and mild menopausal symptoms were comparable between the two groups. Following nine menstrual cycles, the incidence of amenorrhea reached $100 \%$ in the two
\end{abstract}

Correspondence to: Dr Shu-Wen Wang, Department of Radiation Oncology, The First Affiliated Hospital of Medical College of Xi'an Jiaotong University, No. 277 Yanta West Road, Xi'an, Shaanxi 710061, P.R. China

E-mail: wenshuwang2012@163.com

Key words: ovarian castration, high-intensity focused ultrasound, radiotherapy, breast cancer groups. HIFU therapy is superior to radiotherapy for ovarian castration in premenopausal females with $\mathrm{ER}^{+} / \mathrm{PR}^{+}$breast cancer subsequent to radical mastectomy in terms of its minimal invasiveness and faster efficacy. HIFU represents a feasible non-surgical approach for ovarian castration.

\section{Introduction}

Breast cancer is one of the most common malignancies among females and represents an enormous public health problem worldwide. Although the full etiology of breast cancer remains to be elucidated, there is substantial evidence that breast cancer risk is greatly affected by endogenous ovarian hormones (1). Endocrine therapy is the most effective form of systemic therapy for breast cancer, particularly in premenopausal females with hormone receptor-positive breast cancer (2). Ovarian ablation or suppression, which reduces or eliminates endogenous estrogen production, has a beneficial effect on reducing the rates of recurrence, metastasis and mortality in premenopausal females with breast cancer following radical surgery (3).

Ovarian ablation has been used to treat breast cancer for more than 100 years and is achieved by oophorectomy or irradiation of the ovaries $(4,5)$. Oophorectomy reliably and promptly reduces circulating estrogen levels to postmenopausal levels in almost all patients but requires hospitalization and carries potential surgery- and anesthesia-related morbidity and mortality $(6,7)$. The ovarian irradiation procedure is easy and inexpensive, but the procedure does not consistently produce a fall in circulating levels of estrogens (6). Furthermore, a slower rate of estrogen decline and the potential long-term toxicities of pelvic irradiation limit the utility of this approach $(7,8)$. Thus, the development of new minimally invasive castration approaches that enable the rapid and consistent reduction of circulating estrogen levels has great clinical significance in the treatment of breast cancer.

High-intensity focused ultrasound (HIFU) is a relatively new and non-invasive technology that utilizes ultrasound waves focused onto an extremely small area to generate intense heat. These ablate and destroy pathogenic tissue rapidly. As a surgical tool, HIFU has been used for the management of tumors of the liver, kidney, breast, bone, uterus and pancreas 
and uterine fibroids, in addition to various brain disorders, conduction defects in the heart, surgical hemostasis and for the relief of chronic pain of a malignant origin (9-11). Currently, HIFU is most extensively used to treat localized prostate cancer and to ablate the entire prostate gland. However, there are few studies concerning the application of HIFU to ovarian castration in females with breast cancer.

In the present study, a prospective randomized controlled study was conducted to determine the feasibility, efficacy and safety of HIFU for ovarian castration in 88 patients with estrogen receptor $(\mathrm{ER})^{+} /$progesterone receptor $(\mathrm{PR})^{+}$breast cancer subsequent to radical mastectomy. The use of HIFU was compared with radiation treatment in terms of changes in serum levels of estradiol and estrone and the incidence of menopausal symptoms and amenorrhea. The results suggest that HIFU therapy provides a feasible non-surgical approach for ovarian castration in premenopausal females with breast cancer.

\section{Patients and methods}

Patients. This was a prospective randomized controlled study, which included 88 premenopausal females with pathologically confirmed breast cancer subsequent to radical mastectomy. All patients had undergone six cycles of anthracycline-containing chemotherapy. A total of 38 patients had stage II disease and 50 patients had stage III disease. All patients had ER/ PR-positive tumors, axillary nodal involvement and a Karnofsky score of $\geq 90$. Their age ranged from 34 to 52, with a median age of 42.8 years. These patients were randomly divided into two groups that received either HIFU therapy or radiation. Age, clinical stage and Karnofsky score were comparable between the two groups. The Ethics Committee of the First Affiliated Hospital of the Medical College of Xi'an Jiaotong University, China, approved the study. Written informed consent was obtained from each subject prior to their enrollment in the study.

HIFU procedure. The RDS-HIFU tumor therapy system (RDS, Beijing, China) was used with the following settings: ultrasound frequency $=1 \mathrm{MHz}$, focal region $=1.5 \times 6 \mathrm{~mm}$, sound intensity $=0.9-1.5 \mathrm{~kW} / \mathrm{m}^{2}$, depth $=10-170 \mathrm{~mm}$, pulse $=30$ and pulse width $=300 \mathrm{msec}$. The gastrointestinal tract was routinely cleared of stool and gas during the day prior to the procedure. Conventional B-mode ultrasound examination was performed to project the ovaries onto the body surface in order to define the region of interest (ROI). The patients were placed in a prone position and perfused with degassed water $\left(38^{\circ} \mathrm{C}\right)$, which acted as a coupling medium for the HIFU. Immediately prior to HIFU therapy, an ultrasound examination was repeated to confirm the ROI. HIFU therapy was applied twice daily for 5 days.

Irradiation procedure. Irradiation fields $(6-8 \times 12-16 \mathrm{~cm})$ were designed by projecting the ovaries onto the body surface one week following menstruation. Conventional B-mode ultrasound examinations were performed to confirm whether the ovaries were located within the irradiation fields. Radiation was then delivered to a total prescribed dose of $\mathrm{D}_{\mathrm{T}} 18 \mathrm{~Gy}$ in nine fractions over 11 days.
Outcome measures and safety evaluation. Outcome measures included serum levels of estradiol and estrone, an assessment of the Kupperman index and the presence of secondary amenorrhea. Serum levels of estradiol and estrone were measured using a commercial ELISA kit (Sigma, St. Louis, MO, USA) prior to treatment (1 week following menstruation) and every month following treatment. The Kupperman index was used to assess the severity of overall menopausal symptoms by assessing the severity of 11 menopausal symptoms (hot flushes, paresthesia, insomnia, nervousness, melancholia, vertigo, weakness, arthralgia or myalgia, headache, palpitations and formication). Each symptom on the Kupperman index was rated on a scale from 0 to 3 for no, slight, moderate and severe complaints, respectively. The Kupperman index was calculated by summating the severity of each symptom, multiplied by its weighting factor. Based on the Kupperman index, the patients were divided into three groups: mild, moderate and severe. Secondary amenorrhea was defined as the absence of menstrual periods for three consecutive cycles. In addition, adverse events were monitored and recorded. All patients were followed up for 12 months.

Statistical analysis. Statistical analysis was performed using SPSS 13.0 (SPSS, Inc., Chicago, IL, USA). Continuous variables are expressed as mean \pm standard deviation (SD). Comparisons of serum levels of estradiol and estrone pre- and post-treatment were performed using Student's t-tests. Age, clinical stage, Karnofsky score and the distribution of patients with severe, moderate and mild menopausal symptoms were compared using the Chi-square test. $\mathrm{P}<0.05$ was considered to indicate a statistically significant result.

\section{Results}

Changes in serum levels of estradiol and estrone following treatment. As serum levels of estradiol and estrone primarily reflect the activity of the ovaries during the menstrual cycle, the dynamic changes in serum levels of estradiol and estrone were monitored in patients prior to and following treatment. As shown in Tables I and II, the serum levels of estradiol and estrone were comparable prior to treatment between the HIFU and radiation treatment groups. One month following treatment, serum levels of estradiol and estrone were significantly decreased in the two groups; however, a more significant decline was observed in the HIFU treatment group $(\mathrm{P}<0.05)$. HIFU therapy reduced the levels of circulating estrogens to postmenopausal levels at 1 month, while this effect was achieved 3 months following radiotherapy. Taken together, these data suggest that HIFU therapy reduced the levels of circulating estrogens more rapidly than radiotherapy in premenopausal females with breast cancer following radical mastectomy.

Evaluation of the severity of menopausal symptoms. Following ovarian castration, the cyclical changes in ovarian hormones were lost. As a result, menopausal syndrome or postcastration syndrome may develop. Therefore, the severity of menopausal symptoms was evaluated using the Kupperman index in the two groups of patients. As shown in Table III, more patients developed severe menopausal symptoms in the HIFU therapy 
Table I. Serum levels of estradiol (pmmol/l) prior to and following treatment.

Following treatment

\begin{tabular}{|c|c|c|c|c|c|}
\hline Group & Patients (n) & Prior to treatment & One month & Three months & One year \\
\hline HIFU & 44 & $235.6 \pm 36.4$ & $127.1 \pm 28.4^{\mathrm{a}}$ & $118.6 \pm 14.9^{a}$ & $100.6 \pm 23.1^{\mathrm{a}}$ \\
\hline RT & 44 & $233.5 \pm 45.3$ & $191.4 \pm 37.1^{\mathrm{b}}$ & $120.1 \pm 13.2^{\mathrm{a}}$ & $95.7 \pm 12.5^{\mathrm{a}}$ \\
\hline
\end{tabular}

Data shown are expressed as mean $\pm \mathrm{SD}$. ${ }^{\mathrm{P}} \mathrm{P}<0.01$ vs. prior to treatment; ${ }^{\mathrm{b}} \mathrm{P}<0.05$ vs. prior to treatment. HIFU, high-intensity focused ultrasound; RT, radiotherapy.

Table II. Changes in serum levels of estradiol (pmmol/l) between prior to and following treatment.

Following treatment

\begin{tabular}{|c|c|c|c|c|c|}
\hline \multirow[b]{2}{*}{ Group } & \multirow[b]{2}{*}{ Patients (n) } & \multirow[b]{2}{*}{ Prior to treatment } & \\
\hline & & & One month & Three months & One year \\
\hline HIFU & 44 & $221.5 \pm 37.6$ & $63.2 \pm 22.4^{\mathrm{a}}$ & $54.3 \pm 10.4^{\mathrm{a}}$ & $51.4 \pm 7.9^{\mathrm{a}}$ \\
\hline RT & 44 & $212.4 \pm 43.1$ & $102.4 \pm 17.8^{\mathrm{b}}$ & $59.8 \pm 15.3^{\mathrm{a}}$ & $52.3 \pm 9.3^{\mathrm{a}}$ \\
\hline
\end{tabular}

Data shown are expressed as mean $\pm \mathrm{SD}$. ${ }^{\mathrm{P}} \mathrm{P}<0.01$ vs. prior to treatment; ${ }^{\mathrm{b}} \mathrm{P}<0.05$ vs. prior to treatment. HIFU, high-intensity focused ultrasound; RT, radiotherapy.

Table III. Evaluation of the severity of menopausal symptoms in patients after treatment.

\begin{tabular}{lccccccc}
\hline & & \multicolumn{2}{c}{ One month following treatment } & & \multicolumn{2}{c}{ Three months following treatment } \\
\cline { 3 - 6 } Group & Patients (n) & Mild & Moderate & Severe & & Mild & Moderate \\
\hline HIFU & 44 & 6 & 11 & 27 & 3 & 16 \\
RT & 44 & $25^{\mathrm{a}}$ & $15^{\mathrm{a}}$ & $4^{\mathrm{a}}$ & & $5^{\mathrm{b}}$ & 25 \\
\hline
\end{tabular}

${ }^{\mathrm{a}} \mathrm{P}<0.01$ vs. HIFU therapy; ${ }^{\mathrm{P}}>0.05$ vs. HIFU therapy. HIFU, high-intensity focused ultrasound; RT, radiotherapy.

group than in the radiotherapy group 1 month following treatment. Three months following treatment, the distribution of patients with severe, moderate and mild menopausal symptoms was comparable between the two groups.

Incidence of amenorrhea following treatment. Successful ovarian castration invariably results in the presence of amenorrhea. Therefore, the incidence of amenorrhea in patients subsequent to treatment by either HIFU or radiotherapy was evaluated. In the first menstrual cycle following treatment, the menstrual period occurred in five patients in the radiation group, but stopped in all patients in the HIFU therapy group. Following nine menstrual cycles, the incidence of amenorrhea was $100 \%$ in the two groups. Collectively, these data suggest that HIFU therapy resulted in the earlier presence of amenorrhea than radiotherapy in premenopausal females with breast cancer following radical mastectomy.

Adverse effects. A total of five patients developed skin blistering following HIFU therapy, which recovered following conservative treatment. No severe complications, including intestinal perforation, hemorrhage, severe thermal injury or multiple organ dysfunction or failure, occurred in either group.

\section{Discussion}

Previous studies have demonstrated that ovarian castration delays tumor recurrence, reduces metastasis and improves mortality in premenopausal patients with $\mathrm{ER}^{+} / \mathrm{PR}^{+}$breast cancer following radical mastectomy (3). Oophorectomy and irradiation of the ovaries are two approaches commonly used for ovarian castration; however, they are either invasive or have a higher failure rate (6). HIFU is a potential method for the management of localized lesions and provides the only non-invasive means by which deep tissues may be targeted. It is not only applicable to benign lesions but also to a number of malignant lesions. With the advantage of minimal invasiveness, HIFU therapy has an appreciable clinical significance for ovarian castration in patients with breast cancer. The present study compared the efficacy of HIFU therapy versus radiotherapy for ovarian castration in patients with $\mathrm{ER}^{+} / \mathrm{PR}^{+}$breast cancer following radical mastectomy. It was demonstrated 
that HIFU therapy reduced the levels of circulating estrogens more rapidly and was associated with the earlier presence of more severe menopausal symptoms and amenorrhea than radiotherapy.

In contrast to hyperthermia therapy, which heats tissues much less rapidly and to much lower therapeutic temperatures, HIFU therapy permits the generation of extremely high temperatures $\left(70-100^{\circ} \mathrm{C}\right)$ that may be instantly focused on target tissues, rapidly destroying them, without damaging intervening tissue or cells (12). HIFU therapy destroys local tissues mainly through inducing coagulative necrosis. As coagulative necrosis is confined to the treated region, this technology achieves the precise ablation of diseased tissue. In addition, although HIFU treatment may severely damage small blood vessels with the cessation of blood flow, it has no significant impact on large blood vessels near the target tissue $(13,14)$. Instead, HIFU is effective in producing hemostasis (15-17). Therefore, HIFU is a safe method for tissue ablation.

HIFU has been successfully applied in the management of a wide variety of benign and malignant solid lesions (9-11). Wang and Sun demonstrated that HIFU was effective in destroying tumor tissue, alleviating abdominal pain and enhancing cell-mediated immunity in patients with late-stage pancreatic carcinoma (18). Köhrmann et al demonstrated that thermal ablation of renal carcinomas was achieved by HIFU (19). Chan et al demonstrated that HIFU induced the necrosis of uterine fibroids (20).

In previous years, certain researchers have attempted to examine the use of HIFU as an alternative to certain surgeries for the ablation of specific tissues or organs. Roberts et al indicated that HIFU is feasible for the ablation of the vas deferens in a canine model $(21,22)$. Köhrmann et al revealed that HIFU induces clear necrosis in parenchymal tissue and has a use in the therapeutic non-invasive ablation of deep tissues (23). Persson et al demonstrated that it is possible to heat the nucleus pulposus of intervertebral discs by HIFU for the treatment of lower back pain (24). These findings suggest the feasibility of using HIFU to ablate tissues or organs precisely.

The ovaries are extremely sensitive to radiation and the exposure of the ovaries to a radiation dose of $\mathrm{D}_{\mathrm{T}} 18 \mathrm{~Gy}$ or more results in irreversible infertility (25). Radiation progressively induces apoptosis, tissue fibrosis and eventually organ atrophy. Previous studies have shown that the levels of circulating steroid hormones began to decline 2 weeks following radiotherapy (25). The present study demonstrated that serum estradiol and estrone levels were decreased significantly 1 month following radiotherapy and reached postmenopausal levels at 3 months. Unexpectedly, serum estradiol and estrone levels remained low at 1 year post-treatment. In contrast to radiotherapy, HIFU therapy induces massive necrosis of the target tissue and the levels of circulating steroid hormones often begin to decline several hours subsequent to therapy. Accordingly, it was observed that serum estradiol and estrone levels decreased to postmenopausal levels at 1 month following HIFU therapy. These data suggest that the effects of surgical castration are achieved by HIFU therapy.

An evaluation of the incidence of amenorrhea and the severity of menopausal symptoms also highlights the improved efficacy of HIFU therapy compared with ovarian radiotherapy for ovarian castration. The incidence of amenorrhea at 1 month following treatment was significantly higher in the HIFU therapy group than in the radiotherapy group. In addition, although the distribution of patients with severe, moderate and mild menopausal symptoms was comparable at 3 months in patients treated by HIFU and those by radiation, more patients developed severe menopausal symptoms in the HIFU therapy group 1 month following treatment. We speculate that these variations were due to an asynchronous decline in circulating hormones between the two groups of patients.

HIFU is a localized therapy and only treats lesions within a limited area. Normally, it cannot treat a condition that has spread throughout the body. When a disease is widespread, systemic therapies, including hormonal therapy must be applied. However, as the ovary is an endocrine organ, HIFU-mediated ovarian castration provides an effective systematic therapy for females with breast cancer. In addition, HIFU therapy induces coagulative necrosis in solid organs or lesions and it is difficult to identify necrotic tissue and evaluate therapeutic efficacy. Multi-point puncture biopsy is hard to perform and often causes further damage. As serum levels of estradiol and estrone reflect the changes in ovarian function sensitively, they may be used to evaluate the response to HIFU-mediated castration. This offers an extremely convenient method for optimal HIFU therapeutic parameters to be identified. Thus, the ovary may be a suitable target for HIFU therapy.

The adverse effects of HIFU therapy must also be considered. HIFU exposure may cause vessel spasm and hemorrhage when transient cavitation is present and this condition should be avoided during therapy (16). Moreover, skin toxicities are commonly associated adverse events. In the present study, no side effects were observed subsequent to HIFU therapy, with the exception of skin blisters. In addition, as the presence of menopausal symptoms occur earlier after HIFU therapy, appropriate management strategies should be adopted to overcome this problem.

In conclusion, the present study demonstrates that HIFU therapy is superior to ovarian radiation for ovarian castration in premenopausal females with $\mathrm{ER}^{+} / \mathrm{PR}^{+}$breast cancer following radical mastectomy in terms of its minimal invasiveness and faster efficacy. However, the earlier presence of menopausal symptoms following HIFU therapy stresses the necessity for appropriate management measures. Taken together, these results suggest that HIFU therapy provides a feasible non-surgical approach for ovarian castration.

\section{Acknowledgements}

We thank Medjaden Bioscience Limited (Beijing, China) for assisting in the preparation of this manuscript.

\section{References}

1. Eliassen AH and Hankinson SE: Endogenous hormone levels and risk of breast, endometrial and ovarian cancers: prospective studies. Adv Exp Med Biol 630: 148-165, 2008.

2. Pritchard K: Endocrinology and hormone therapy in breast cancer: endocrine therapy in premenopausal women. Breast Cancer Res 7: 70-76, 2005.

3. Colleoni M and Giobbie-Hurder A: Benefits and adverse effects of endocrine therapy. Ann Oncol 21 (Suppl 7): vii107-vii111, 2010 . 
4. No authors listed: Ovarian ablation in early breast cancer overview of the randomised trials. Early Breast Cancer Trialists Collaborative Group. Lancet 348: 1189-1196, 1996.

5. Ravdin RG, Lewison EF, Slack NH, et al: Results of a clinical trial concerning the worth of prophylactic oophorectomy for breast carcinoma. Surg Gynecol Obstet 131: 1055-1064, 1970.

6. Prowell TM and Davidson NE: What is the role of ovarian ablation in the management of primary and metastatic breast cancer today? Oncologist 9: 507-517, 2004.

7. Davidson NE: Ovarian ablation as treatment for young women with breast cancer. J Natl Cancer Inst Monogr 16: 95-99, 1994.

8. Dees EC and Davidson NE: Ovarian ablation as adjuvant therapy for breast cancer. Semin Oncol 28: 322-331, 2001.

9. Kennedy JE, Ter Haar GR and Cranston D: High intensity focused ultrasound: surgery of the future? Brit J Radiol 76: 590-599, 2003

10. Stewart EA, Gostout B, Rabinovici J, Kim HS, Regan L and Tempany CM: Sustained relief of leiomyoma symptoms by using focused ultrasound surgery. Obstet Gynecol 110: 279-287, 2007.

11. Wu F, Wang ZB, Cao YD, Chen WZ, Bai J, Zou JZ and Zhu H A randomised clinical trial of high-intensity focused ultrasound ablation for the treatment of patients with localised breast cancer. Br J Cancer 89: 2227-2233, 2003.

12. Oosterhof GO, Cornel EB, Smits GA, Debruyne FM and Schalken JA: Influence of high-intensity focused ultrasound on the development of metastases. Eur Urol 32: 91-95, 1997.

13. Hynynen K, Chung AH, Colucci V and Jolesz FA: Potential adverse effects of high-intensity focused ultrasound exposure on blood vessels in vivo. Ultrasound Med Biol 22: 193-201, 1996.

14. Wu F, Chen WZ, Bai J, Zou JZ, Wang ZL, Zhu H and Wang ZB: Tumor vessel destruction resulting from high-intensity focused ultrasound in patients with solid malignancies. Ultrasound Med Biol 28: 535-542, 2002.

15. Pritchard K: Endocrinology and hormone therapy in breast cancer: endocrine therapy in premenopausal women. Breast Cancer Res 7: 70-76, 2005
16. Hynynen K, Colucci V, Chung A and Jolesz F: Noninvasive arterial occlusion using MRI-guided focused ultrasound. Ultrasound Med Biol 22: 1071-1077, 1996.

17. Noble ML, Vaezy S, Keshavarzi A, Paun M, Prokop AF, Chi EY, Cornejo C, Sharar SR, Jurkovich GJ, Martin RW and Crum LA: Spleen hemostasis using high-intensity ultrasound: survival and healing. J Trauma 53: 1115-1120, 2002.

18. Wang X and Sun J: High-intensity focused ultrasound in patients with late-stage pancreatic carcinoma. Chin Med J (Engl) 115: $1332-1335,2002$

19. Köhrmann KU, Michel MS, Gaa J, Marlinghaus E and Alken P: High intensity focused ultrasound as noninvasive therapy for multilocal renal cell carcinoma: case study and review of the literature. J Urol 167: 2397-2403, 2002.

20. Chan AH, Fujimoto VY, Moore DE, Martin RW and Vaezy S: An image-guided high intensity focused ultrasound device for uterine fibroids treatment. Med Phys 29: 2611-2620, 2002.

21. Roberts WW, Wright EJ, Fried NM, Nicol T, Jarrett TW, Kavoussi LR and Solomon SB: High-intensity focused ultrasound ablation of the epididymis in a canine model: a potential alternative to vasectomy. J Endourol 16: 621-625, 2002.

22. Roberts WW, Chan DY, Fried NM, Wright EJ, Nicol T, Jarrett TW, Kavoussi LR and Solomon SB: High-intensity focused ultrasound ablation of the vas deferens in a canine model. J Urol 167: 2613-2617, 2002.

23. Köhrmann KU, Michel MS, Steidler A, Marlinghaus E, Kraut O and Alken P: Technical characterization of an ultrasound source for noninvasive thermo-ablation by high-intensity focused ultrasound. BJU Int 90: 248-252, 2002.

24. Persson J, Stromqvist B, Zanoli G, et al: Ultrasound nucleolysis: an in vitro study. Ultrasound Med Biol 28: 1189-1197, 2002.

25. Li Y-X and Yu C-X (eds): Practical Gynecologic Endocrinology. Shanghai Medical University Press, Shanghai, pp64-79, 1997. 